\title{
Proximity Capacitance Array Sensor Based on Data Aggregation Strategy for Rainfall Detection
}

\author{
Pengzhan Chen ${ }^{*}$ and Qingqing Yu \\ School of Electrical and Automation Engineering, East China Jiaotong University, \\ Changbei Open and Developing District, Nanchang, Jiangxi 330013, China
}

(Received September 19, 2017; accepted January 31, 2018)

Keywords: rainfall detection, proximity capacitive sensor, sensor array, data aggregation

People need to measure rainfall accurately in many cases, and hence improving the accuracy and real-time performance of rainfall sensors is a current problem. A new type of rainfall sensor based on a proximity capacitance array is proposed. The sensor can be used to detect the rainfall coverage of an insulator surface accurately and efficiently. According to the different characteristics of dielectric constants of different materials, the water film thickness on the surface of the insulator is measured by using the principle of proximity capacitance, and a comprehensive evaluation index of sensor performance is proposed. The finite element method is utilized to compare the characteristics of capacitive sensors using three types of electrode to improve sensor penetrability and achieve high water film detection sensitivity. An electrode array is designed according to optimal results, and multielectrode measurement data are aggregated to determine the amount of rainfall. Moreover, an experimental sensor platform is constructed to verify the proposed rain sensor based on a proximity capacitance array. The designed rainfall sensor possesses good accuracy, and the measurement error is about $\pm 2.3 \%$. The proposed rainfall sensor allows for accurate real-time measurements of rainfall on insulator surfaces with a certain thickness.

\section{Introduction}

Rainfall is a commonly occurring natural phenomenon, and accurately predicting the volume of rainfall in real time is impossible. Sudden rain may negatively affect human life or agricultural production and could even cause loss of life. If rain falls and the windows of houses have not been closed, rain can lead to property damage. If a factory lacks protection against sudden rain, water can damage equipment, and such damage can lead to operation paralysis. Moreover, mitigating the dangerous effects of resulting landslides and overflowing reservoirs becomes difficult when forestry departments fail to obtain real-time rainfall data. Therefore, obtaining real-time and accurate data on the duration and amount of rainfall is crucial in maintaining the quality of human life and the integrity of many production forms. Using rainfall sensors to monitor the rainfall process can provide a basis for alertness and protection,

*Corresponding author: e-mail: cyxcpz@163.com

http://dx.doi.org/10.18494/SAM.2018.1744 
which can reduce or even avoid various losses caused by rainfall. Many rain sensors at present are keyed to specific applications. Rain sensors are classified into five types depending on the general scientific principles adopted, namely, photoelectric, piezoelectric, thermocouple, image, and capacitance. A photoelectric rainfall sensor can realize noncontact detection, but it depends largely on light and demonstrates poor anti-interference capability. ${ }^{(1)}$ A piezoelectric rainfall sensor is susceptible to interference from moisture, and its sensitivity is difficult to control. $^{(2)}$ Meanwhile, the temperature change of the thermocouple rainfall sensor caused by rain is not apparent. ${ }^{(3,4)}$ The sensor is easily affected by changes in environmental temperature and easily corroded by rainwater. Image rainfall sensors based on image-processing technology are vulnerable to external physical interference, and their algorithms are complex and costly. ${ }^{(5)}$ These sensors are unsuitable for popularization and difficult to apply because they are generally configured on high-end, specialized equipment. ${ }^{(6,7)}$

Capacitive sensors have been widely used in various fields because of their high sensitivity, fast dynamic response, and low cost. ${ }^{(8-10)}$ A capacitive sensor does not need to be in physical contact with the object to be measured during the measurement process and is unaffected by light, vibration, and other external environmental factors. Therefore, researchers use the capacitance principle to design rainfall sensors. ${ }^{(11-13)}$ The principle underlying capacitive sensors is based on the different characteristics of the dielectric constants of different materials. ${ }^{(14)}$ However, current capacitive rain sensors cannot provide an accurate assessment of rainfall amounts in real time, and their application is limited. In this study, we developed an optimized capacitive sensor to solve current problems.

\subsection{Previous studies}

The problem of improving the accuracy of rainfall detection by using capacitive sensors has been discussed in many studies. ${ }^{(15-19)}$ Several scholars have optimized capacitive rainfall sensors. In 2010, Nan Li et al. developed a new proximity capacitive sensor design for an automatic car wiper control system, but the authors did not conduct follow-up research on the measurement system, and no sensor application was demonstrated. ${ }^{(15)}$ In practice, the output characteristic of a sensor has difficulty meeting the linear relationship because of the influence of parasitic capacitance and the edge effect. ${ }^{(16)}$ In 2016, Long Zhang et al. proposed a method of reducing measurement errors by using the backpropagation (BP) neural network to fit the capacitive sensor output characteristic curves, but the scheme requires a large amount of calibration data as training samples to establish the model; this requirement leads to low learning speed and complex calculation. Baopeng Lai et al. designed a cylindrical capacitive rain sensor, but the sensor is large and cannot be used in many practical applications. ${ }^{(17)}$ Realtime performance cannot be guaranteed because the container structure leads to uneven liquid density, which affects the measurement result. In summary, the methods proposed in these studies address the problem to some extent but cause other issues, such as increased computational complexity, limited practical application, and poor real-time performance.

To address the complexity and practical limitations of existing solutions, we designed a scheme for a rainfall sensor based on the principle of proximity capacitance. The measurement accuracy of the sensor and the thickness of medium penetration were improved by optimizing 
the capacitor electrode. The data aggregation algorithm of array sensors was introduced in the measurement scheme to ensure the real-time performance and accuracy of the rainfall measurement process. The proposed sensor possesses high accuracy and is inexpensive and easy to fabricate. In addition, the sensor can be installed inside or below the insulator and is thus not easily affected by environmental factors during measurement. These sensor features facilitate real-time and high-precision rainfall measurement.

\section{Optimization of Capacitive Sensor Electrodes}

\subsection{Principle and performance index}

The measurement principle of proximity capacitance is to attach two proximity electrodes to the inner surface of a probe body of a certain thickness to form a stable electrostatic field through the edge effect of the capacitor plates. When the material in the electric field changes, the relative permittivity between the two electrode plates changes and leads to a change in the capacitance. The variation in the material in the electric field can be obtained by measuring the capacitance because the dielectric constants of the medium, air, and rainwater are different. When rain falls on the surface of the object, the capacitance of the electrode plate changes depending on the thickness of the rainwater. The measurement system can accurately determine the presence of rainwater and film size by measuring the variation in capacitance between the plates. The measurement principle is illustrated in Fig. 1.

The relationship between the capacitance of a parallel plate capacitor and the liquid capacitance distribution is provided by the equation

$$
C=-\frac{1}{V} \iint_{\Gamma} \varepsilon_{0} \varepsilon(s) \nabla \phi(s) d \Gamma
$$

where $\varepsilon(s)$ indicates the permittivity in the detection area of the capacitive sensor, $\varepsilon_{0}$ indicates the constant, $V$ indicates the electric potential difference between adjacent plates, $\phi(s)$ indicates

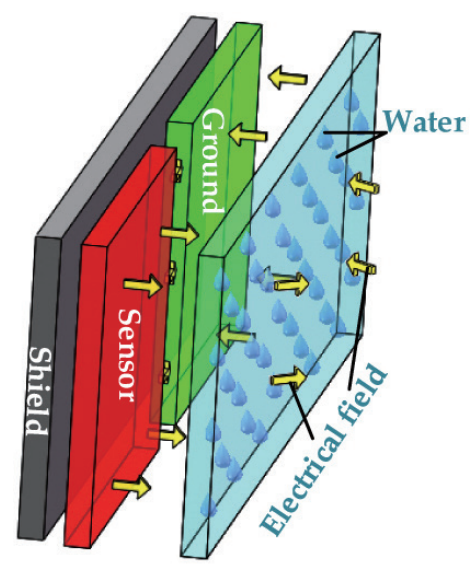

Fig. 1. (Color online) Measurement principle of adjacent capacitive sensor. 
the potential distribution, and $\Gamma$ indicates the surface of the electrode plate. According to the analysis above, the capacitance of the proximity capacitive sensor is closely related to (1) the equivalent area of the sensor plate, (2) the equivalent interval between the excitation and receiving electrodes, and (3) the relative permittivity of objects to be detected in the detection area, detection objects, and air. When rainfall measurement is conducted according to the principle of proximity capacitance, the capacitance in the detection area is the result of the interaction of rain, air, and the sensor, in addition to the effects of temperature and possible contamination with dirt. When the water film thickness on the surface of the medium changes, a change in the capacitance rate of the capacitive sensor results in a change in the sensor capacitance because the compositions of the medium, air, and sensor are basically similar.

Penetration depth, signal intensity, and sensitivity are important performance indices of adjacent capacitive rain sensors. The penetration depth of an adjacent capacitive sensor theoretically defines an effective measurement, that is, the decay rate and attenuation of the electric field measured by the sensor caused by the increase in the distance between the object and the sensor. ${ }^{(20)}$ This index is used to evaluate the measurement intensity and sensor sensitivity, determine the thickness that the electric field lines can penetrate, and obtain the thickness of the insulating film layer on the surface of the measured object. The penetration depth of a capacitive sensor is estimated by experimental measurement. The signal intensity, namely the size of the capacitance, can directly determine the minimum signal resolution of the system and affect the measurement sensitivity. The design must be optimized to increase the output signal intensity because the measured edge capacitance of an adjacent capacitive sensor is very low (usually $\mathrm{pF}$ ). The external circuit requires a high resolution. To avoid distortion, the effect of sensor noise must be mitigated by adding a shielding layer to minimize noise. ${ }^{(21)}$ Different measurement sensitivities of different locations of the sensor sensing area and different distances with the sensor surface are obtained owing to the inhomogeneity of the electric field distribution. Reference 22 demonstrated that improving the consistency of electric field distribution is one of the evaluation indices of sensor performance. The slope of the measured curve of the adjacent capacitive sensor is defined as measurement sensitivity at this distance, that is, the ratio between the change in the output value of the sensor and the change in the measured parameters of the sensor. During rainfall measurement, the sensor must ensure that within a certain range, a water film thickness change can cause the sensor capacitances to change in the dry state, thereby enabling the sensor to obtain high sensitivity. Reference 23 indicated that the dynamic range of an adjacent edge capacitive sensor is the ratio of the maximum output to the input value of the sensor and depends mainly on different sensor measurements. The maximum and minimum measurements must be within the measurable range of the external circuit.

Reference 24 showed that the geometric parameters of the electrode exert great influence on sensor performance. The longer the effective edge length and the larger the public area, the stronger the signal. The performance of an interdigital sensor is much better than that of annular and rectangular sensors with a single structure; however, the interdigital sensor cannot meet the measurement demand of the rainfall sensor. ${ }^{(24)}$ Therefore, a new type of electrode was developed in the current study by optimizing the geometrical parameters of the electrode to meet the measurement requirements of the sensor. 


\subsection{Optimization of electrode geometry parameters}

To determine the electrode form that can match the performance of the rain sensor, three electrode schemes for the adjacent electrode capacitive sensor were designed, as shown in Figs. 2(a)-2(c). Optimal results were obtained by comparing the performance of these three types of sensor. Figure 2(a) shows an interdigital sensor. In Fig. 2(b), the resulting multiring structure of the sensor expands the scope of the dynamic measurement capability of the sensor by using several ring plates. The structure of the ice crystal rain sensor [see Fig. 2(c)] is complex and obtains a high edge capacitance by extending the area of the excitation electrode. The symmetrical design improves the consistency of the electric field. The proof of the area of irregular figures can be estimated by counting the square grids or via the integral method.

The performance of a capacitive sensor is closely related to sensor size. We made the sensor sizes of the different electrodes shown in Fig. 2 consistent to objectively compare the electric field characteristics of the proximity capacitive sensors under different electrode schemes, and the overall measurement environment was consistent. We used polychlorinated biphenyl (PCB) to reduce the cost of manufacturing, the thicknesses of the sensors were $1 \mathrm{~mm}$, and the electrodes were made of copper and were $0.2 \mathrm{~mm}$ thick. The peripheral squares were set to $\delta_{1}=52 \mathrm{~mm}$, shielding layers were arranged, and their thicknesses were $0.5 \mathrm{~mm}$. The specific dimensions of the three types of rainfall sensor are presented in Table 1. The optimal design of capacitive sensor electrodes was obtained by comparing the performance of the three electrode schemes.

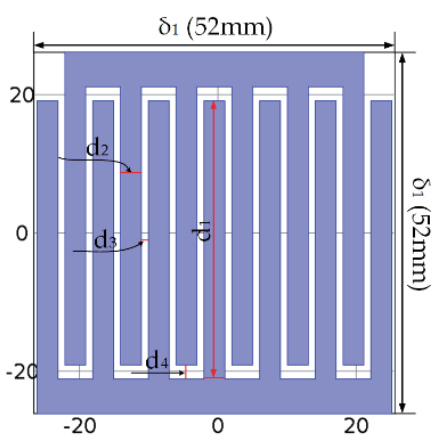

(a)

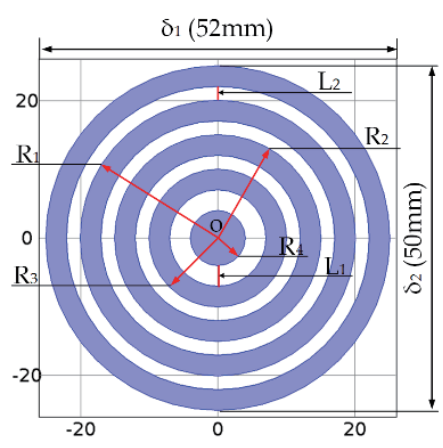

(b)

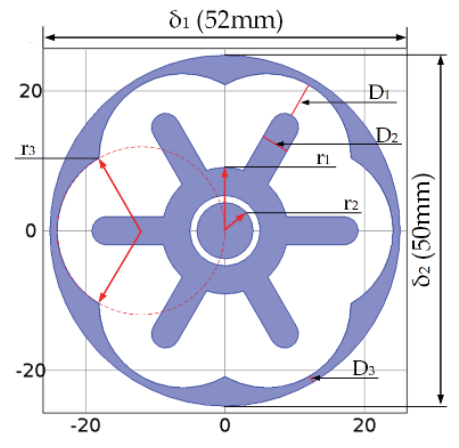

(c)

Fig. 2. (Color online) Geometric structure and size of the three proximity capacitive sensors described: (a) interdigital, interactive form; (b) multiring structure adopting a plurality of circular plates labeled R1-R4 for the maximum radius of each ring (the ring width is $2 \mathrm{~mm}$ ); and (c) ice-crystal-shaped structure, which is complex and has an internal structure similar to that of ice crystals.

Table 1

Specific dimensions of three types of rainfall sensor.

\begin{tabular}{lcccccc}
\hline Sensors & \multicolumn{7}{c}{ Length $(\mathrm{mm})$} \\
\hline Interdigital sensor & $d_{1}$ & $d_{2}$ & $d_{3}$ & $d_{4}$ \\
& 40 & 3 & 1 & 2 & & \\
Multiring sensor & $R_{1}$ & $R_{2}$ & $R_{3}$ & $R_{4}$ & $L_{1}$ & $L_{2}$ \\
& 20 & 15 & 10 & 4 & 3 & 2 \\
Ice crystal sensor & $r_{1}$ & $r_{2}$ & $r_{3}$ & $D_{1}$ & $D_{2}$ & $D_{3}$ \\
& 9 & 4 & 12 & 5 & 4 & 1 \\
\hline
\end{tabular}


The magnitude and distribution of the electric field affect many performance indicators of the proximity capacitive sensor because this sensor performs measurement based on the change in the capacitance ratio in the electric field space formed by the electrode. The basis for evaluating the performance of different electrode types used in sensors can be obtained by analyzing and comparing the electric field characteristics of several types of proximity capacitive sensor. To compare the performance of different types of electrode-based capacitive rain sensor, in this study, we utilized COMSOL software to simulate and analyze the electric field characteristics of all sensor types by measuring the electric field penetration depth, signal strength, and sensor sensitivity.

A simulation experiment was conducted under the condition that the capacitive sensor has a fixed size, and the length and width of the sensors are $52 \mathrm{~mm}$. Excitation voltage was set to $3 \mathrm{~V}$ in consideration of the limited cost, the electrode of the capacitive sensor used in the experiments having a copper plate structure, and the influence of the shielding layer on the measurement. The excitation and receiving electrodes in the simulation were set as follows. In Fig. 2(a), the two upper and lower electrodes of the comb-type capacitive sensor are the receiving and exciting electrodes, respectively. In Fig. 2(b), the center circle of the multiring capacitive sensor is the exciting electrode, and the four concentric rings comprise the receiving electrode. In Fig. 2(c), the crystal structure of the ice-crystal-shaped capacitive sensor is the excitation electrode, and the peripheral part is the receiving electrode. During the simulation, a shielding layer was arranged at the periphery and bottom of each sensor plate to reduce the noise interference affecting the sensor and to focus the penetration direction of the electric field line.

\subsection{Results of the simulation experiment}

The simulation results are based on the Maxwell boundary condition equation. In the process of simulation, the value of the potential on the boundary of the field is given as a second-boundary-value problem or Neumann problem; the finite element method is a natural boundary condition, and the simulation results are shown in Fig. 3. ${ }^{(25)}$

According to the simulation results in Figs. 3(a)-3(c), under the same conditions, the electric field strengths of the three rainfall sensors differed. The electric field intensities of the interdigital and multiring sensors were relatively weak. The electric field intensity of the ice crystal sensor was the largest, the distribution was consistent, and the maximum value was $57.67 \mathrm{~V} / \mathrm{m}$. The analysis results showed that the greater the distance between the receiving and exciting electrodes, the stronger the electric field signal (i.e., the deeper the penetration of the electric field line).

According to the simulation results in Figs. 3(d)-3(f), the potential difference in the multiring sensor near the potential part of the receiving and excitation electrodes was large, whereas the area far from this part was small. Figure 3(e) shows that the maximum voltage change was about $1.56 \mathrm{~V}$. The detection range of the multi-ring sensor was small, and the average capacitance of the sensor was low. The interdigital capacitive sensor voltage change was about $2.52 \mathrm{~V}$, and the potential distribution was consistent. However, Fig. 3(d) shows that the plate potential of the middle part of the sensor was weak (about 0.4-0.6 V). Figure 3(f) shows that 


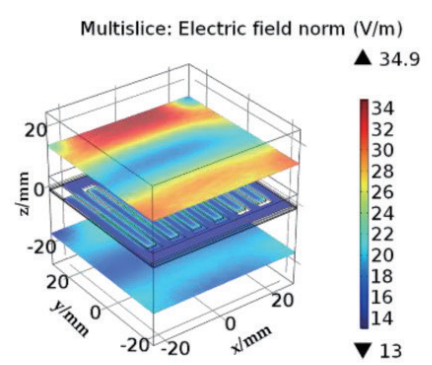

(a)

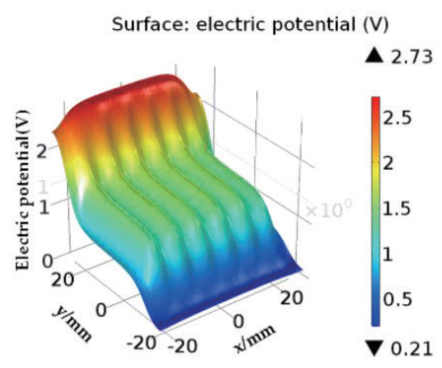

(d)

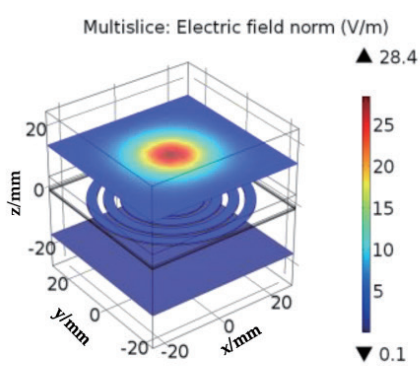

(b)

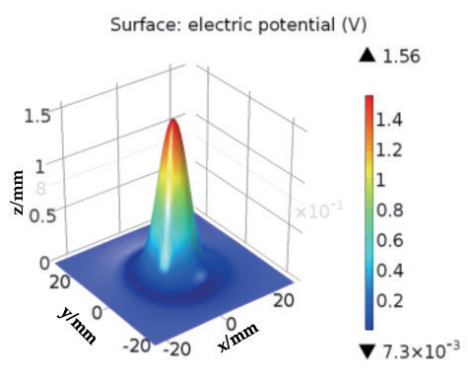

(e)

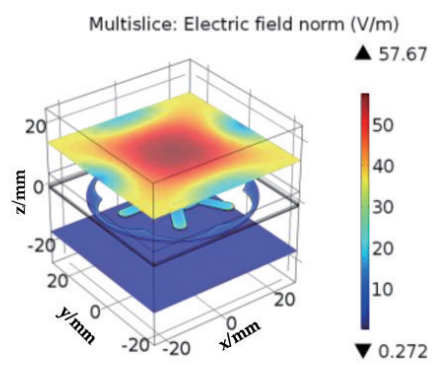

(c)

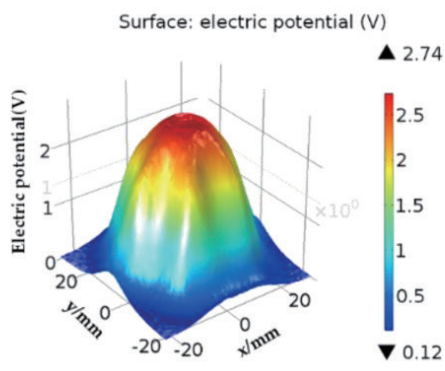

(f)

Fig. 3. (Color online) Electric field intensity distributions of three types of rainfall sensor: (a) interdigital sensor, (b) multiring sensor, and (c) ice crystal sensor. The section of the electric field intensity is given in the figure. The section is obtained from the plane $15 \mathrm{~mm}$ of the $Z$-axis. Potential distribution of three rainfall sensors: (d) interdigital sensor, (e) multiring sensor, and (f) ice crystal sensor. The potential distribution of the sensor on the plane and the simulation of the $Z$-axis at $5 \mathrm{~mm}$ are shown.

the electric potential distribution of the ice crystal sensor was consistent, the voltage was the highest, and the maximum potential was about $2.62 \mathrm{~V}$. The middle part of the potential plate was clear (about $0.5-1.5 \mathrm{~V}$ ). The ice crystal sensor combines the advantages of the interdigital and multiring capacitive sensors, and the area of the exciting electrode and the distance between the plates are increased under the same size and excitation voltage; thus, the edge capacitance is increased, and the sensitivity of the sensor is improved.

The sensitivity of the sensor can directly reflect sensor performance. Therefore, the sensitivities of the different electrode schemes were compared by analyzing the variation in the capacitance when dielectric films were covered with water films of different thicknesses. Given a square acrylic plate of $3.8 \mathrm{~mm}$ thickness and $52 \mathrm{~mm}$ length, we used the capacitances of the three types of sensor electrode to analyze the different thicknesses of the acrylic plate with a water film capacitor cover. The initial state was dry. The initial capacitances of the interdigital, multiring, and ice crystal sensors were 15.624, 1.2284, and $5.2989 \mathrm{pF}$, respectively. The thickness of the water film cover increased from 0.1 to $0.5 \mathrm{~mm}$ in the same detection area. The values of the three sensors were obtained through a COMSOL simulation, as shown in Table 2 .

Figure 4 shows that when the three capacitive sensors were covered with the water film, the fringing capacitance was increased compared with that without the water film. The capacitance of the ice crystal sensor showed the largest percentage increase; however, the increase was 
Table 2

Values of sensors with different water film thicknesses.

\begin{tabular}{lccc}
\hline Water thickness $(\mathrm{mm})$ & Interdigital sensor & Multiring sensor & Ice crystal sensor \\
\hline 0.1 & $15.781^{*}$ & 1.2756 & 5.5554 \\
0.2 & 15.867 & 1.3030 & 5.7478 \\
0.3 & 15.929 & 1.3206 & 5.9016 \\
0.4 & 15.972 & 1.3346 & 6.0288 \\
0.5 & 16.011 & 1.3451 & 6.1358 \\
\hline
\end{tabular}

*True value of fringing capacitance $(\mathrm{pF})$.

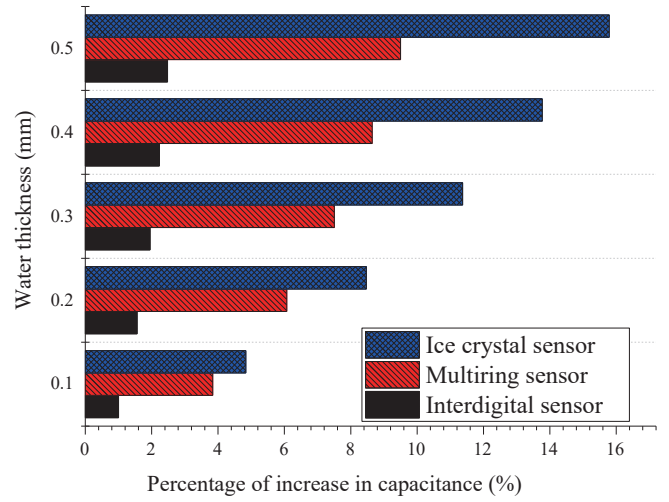

Fig. 4. (Color online) Increasing percentage of sensors with different water film thicknesses.

nonlinear. In addition, the fringing capacitance was different in the initial state because of the different structures of the three capacitive sensors.

According to the abovementioned concept of sensor sensitivity and to better compare the performance of different sensors, the following equation is defined. ${ }^{(26)}$

$$
\varphi_{i}=\frac{\left(C_{i}-C_{a i r}\right) / C_{a i r}}{l_{i}}, i=1,2,3,4,5
$$

In the equation, $\varphi_{i}$ is the measurement sensitivity of the sensor with different water film thicknesses, $C_{\text {air }}$ is the fringing capacitance of the sensor when the sensor is dry and the detection medium is air, $C_{i}$ is the fringing capacitance of the sensor with different water film thicknesses, and $l_{i}$ stands for different water film thicknesses. The slope curves of the three sensors are shown in Fig. 5.

As shown in Fig. 5, the sensitivity of the ice crystal sensor to the thickness of the water film was the highest, and the sensor showed good resolution of spatial permittivity. The slope curve of the sensor decreased with the increase in water film thickness.

In addition, the performances of sensor would be changed depending on the choice of the design parameters, and the comparison results may lead to the discussion of fairness. Therefore, in order to ensure the fairness of the comparison results of these three types of sensor, we have also done a series of simulation experiments. For the interdigital sensor, the gap between the teeth is increased and the number of comb teeth is reduced, for example, when the number of comb teeth of the excitation electrodes are decreased to five, the corresponding gap increased 


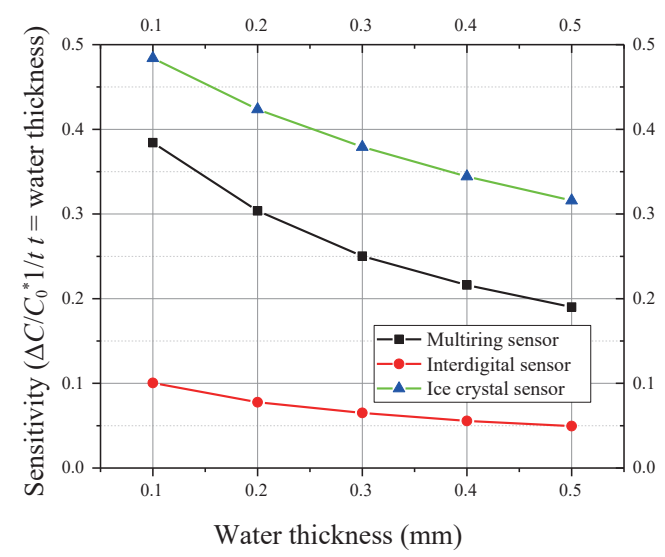

Fig. 5. (Color online) Line graph of the sensitivity differences of the capacitive sensors.

to $1.8 \mathrm{~mm}$. Similarly, the multiring sensor in the experiment to decrease the number of rings, while increasing the gap between the rings. As the shape of the ice crystal sensor is complex, in this experiment, we respectively increase and decrease the size of the internal pattern. The simulation results of the electric field intensity and electric potential difference of the sensors are shown in Table 3.

In Table 3, the electric field intensity of the interdigital and multiring sensors increases as the number of teeth/rings decreases and the gap between the teeth/ring increases, but the increase is very small. Regardless of whether the internal pattern size of the ice crystal sensor is increasing or decreasing, the electric field intensity of the sensor is still the largest. In addition, the change in the electric potential difference of the interdigital sensor is smaller, and the electric potential difference of the multiring sensors increases as the number of rings decreases and the gap between the ring increases, but the increase is very small. The internal pattern size of the ice crystal sensor is increasing, the electric potential difference of the sensor is the largest.

In order to compare the performance of these three types of sensor more directly, the simulation experiment will be continued, and the capacitances of these sensors will be obtained. Then the sensitivities of the sensors under the choice of different parameters are calculated according to Eq. (2). The results are shown in Fig. 6.

As shown in Fig. 6, the ice crystal sensors were compared with the other two types of sensor; the sensitivities of the ice crystal sensors to the thickness of the water film were the highest, and the sensors showed good resolution of spatial permittivity.

A comprehensive comparison of the penetration performance of electric field lines in all types of electrode and measurement sensitivity showed that all performance indices of the ice crystal sensor were excellent, the electric field line penetration performance was strong, the dynamic measurement range was wide, and the sensitivity of the sensor, which directly determines the performance, was much higher than that of the rest. The analysis results showed that the ice crystal capacitive sensor had the best design scheme of the sensor electrode and can meet the requirements of high-sensitivity measurement systems. However, a capacitive sensor with a single structure is sometimes unable to meet the needs of several practical applications. 
Table 3

Electric field intensity and potential difference of the three types of sensor.

\begin{tabular}{|c|c|c|c|c|}
\hline \multicolumn{5}{|c|}{ Electric field intensity (V/m) } \\
\hline \multirow{2}{*}{ Interdigital sensor } & Gap $(1.8 \mathrm{~mm})$ & Gap (3 mm) & Gap (5 mm) & Gap (9 mm) \\
\hline & 33.2 & 33.9 & 36.1 & 36.8 \\
\hline \multirow{2}{*}{ Multiring sensor } & Gap (2 mm) & Gap (4 mm) & Gap $(7.5 \mathrm{~mm})$ & Gap (18 mm) \\
\hline & 28.4 & 29.3 & 32.6 & 34.2 \\
\hline \multirow{2}{*}{ Ice crystal sensor } & Size $(\times 0.5)$ & Size $(\times 1)$ & Size $(\times 1.2)$ & \\
\hline & 52.35 & 57.67 & 53.68 & \\
\hline \multicolumn{5}{|c|}{ Electric potential difference (V) } \\
\hline \multirow{2}{*}{ Interdigital sensor } & Gap $(1.8 \mathrm{~mm})$ & Gap (3 mm) & Gap (5 mm) & Gap (9 mm) \\
\hline & 2.44 & 2.45 & 2.48 & 2.45 \\
\hline \multirow{2}{*}{ Multiring sensor } & Gap (2 mm) & Gap (4 mm) & Gap $(7.5 \mathrm{~mm})$ & Gap $(18 \mathrm{~mm})$ \\
\hline & 1.56 & 1.61 & 1.67 & 1.73 \\
\hline \multirow{2}{*}{ Ice crystal sensor } & Size $(\times 0.5)$ & Size $(\times 1)$ & Size $(\times 1.2)$ & \\
\hline & 2.26 & 2.62 & 2.63 & \\
\hline
\end{tabular}

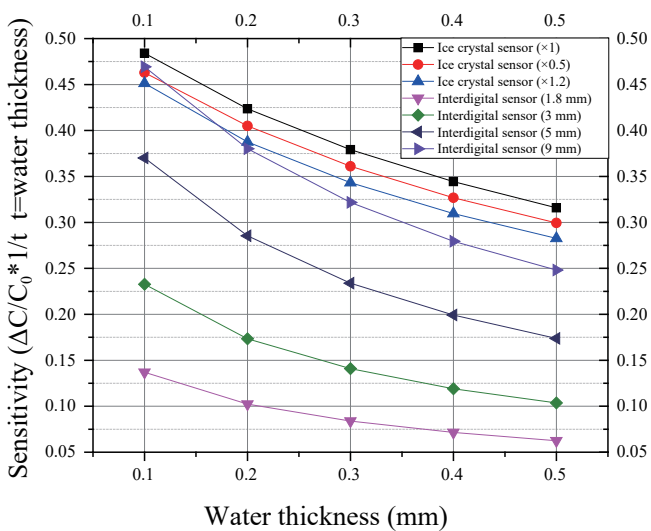

(a)

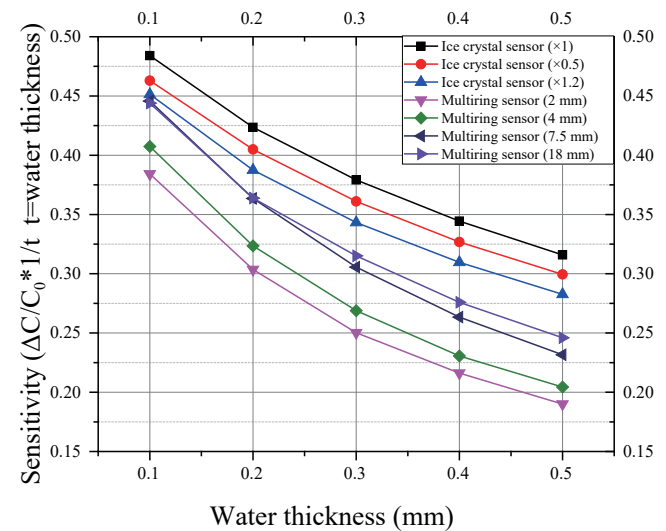

(b)

Fig. 6. (Color online) Line graphs of the sensitivity differences of the capacitive sensors.

\section{Sensor Design and Measurement Strategy}

\subsection{Electrode array design}

The following problems may exist in the practical application of rainfall sensors. In several special applications, the sensor may need to be installed on a tilt angle, it may be occluded by other objects, or the sensor electrode may be worn out by long-term use. In these cases, using a single capacitive sensor for rainfall measurement may produce deviations in the results. Several possible external disturbances were simulated in this study. Figure 7(a) shows the ideal state before the sensor was disturbed. The initial conditions of the simulation were consistent with those of the simulation process, that is, a $3.8-\mathrm{mm}$-thick acrylic plate covering the $0.5 \mathrm{~mm}$ water film. Figure 7(b) shows that when the sensor was shielded in the simulation, the acrylic plate 


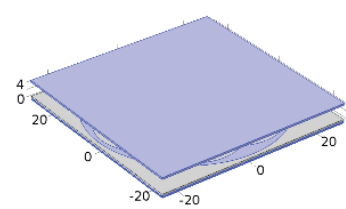

(a)

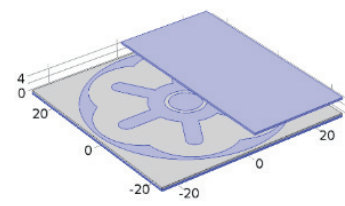

(b)

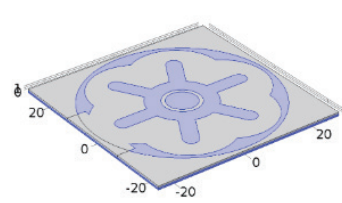

(c)

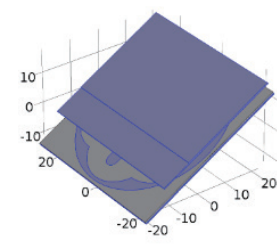

(d)

Fig. 7. (Color online) Different external geometric perturbations of the sensor: (a) ideal initial state of the sensor, (b) sensor occlusion state, (c) sensor electrode wear state, and (d) sensor tilt state.

surface was partly covered by the water film (water film length of $52 \mathrm{~mm}$, width of $26 \mathrm{~mm}$, and thickness of $0.5 \mathrm{~mm}$ ). Figure 7(c) shows the wear condition of the simulated electrode of the sensor, namely, the polarity of the electrode. For convenience, the $0.5 \mathrm{~mm}$ water film and 3.8-mm-thick acrylic plate were set in the hidden mode (a setting in COMSOL software that hides boundaries or entities), which had no effect on the simulation results. Figure 7(d) shows an analog sensor in a tilt condition. The water film distribution was uneven in this initial environment. One part consisted of a $0.5 \mathrm{~mm}$ water film, and the length and width were 52 and $32 \mathrm{~mm}$, respectively. The other part consisted of a $0.3 \mathrm{~mm}$ water film, and the length and width were 52 and $10 \mathrm{~mm}$, respectively. The remaining part has no film covering.

After the simulation environment was built, edge capacitance was obtained by simulation, and Eq. (2) was used in the calculation. The results of the analysis of sensor sensitivity fluctuations and the simulation results are shown in Fig. 8. When the sensor experienced some external geometric interference, its sensitivity changed by varying degrees. This result indicates that a single ice-crystal-shaped capacitive sensor may produce an error in the detection of large amounts of rainfall. Therefore, several electrodes should be used to simultaneously measure the water film thickness on the surface of the sensor. The results of multiple measurements are combined by using the redundant complementary characteristics of multisource information. More reliable measurement results can be obtained with this approach than by using a single measuring electrode.

In the same measurement area (the length of the square area is $104 \mathrm{~mm}$ ), $2 \times 2$ and $4 \times 4$ ice-crystal-shaped sensor array arrangements were designed to accurately measure rainfall according to the results of the optimizing electrode type. The single measurement area of the $2 \times 2$ array sensor was $52 \times 52 \mathrm{~mm}^{2}$, and that of the $4 \times 4$ array sensor was $26 \times 26 \mathrm{~mm}^{2}$. An electric field simulation was carried out (the simulation process is consistent with that mentioned in Sect. 3.1), and the simulation results are shown in Fig. 9.

The voltage variation of the $2 \times 2$ array sensor was about $2.13 \mathrm{~V}$ [Fig. 9(a)], and that of the 4 $\times 4$ array sensor was about $1.22 \mathrm{~V}$ [Fig. 9(b)]. The simulation results in Fig. 9 show that when the single ice crystal sensor area was reduced, the overall voltage of the array sensor decreased, that is, the signal intensity weakened. The sensitivity of the system was analyzed to determine the difference in the performance of the two array sensors. Only the capacitance of two singlesensor components in the $4 \times 4$ array was selected for comparison with that of the $2 \times 2$ array 


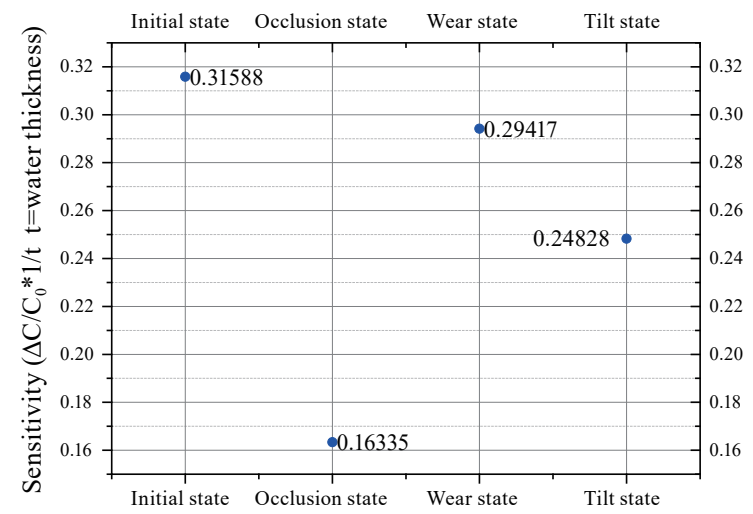

Fig. 8. (Color online) Sensitivity curve of the ice crystal sensor under different external disturbances.

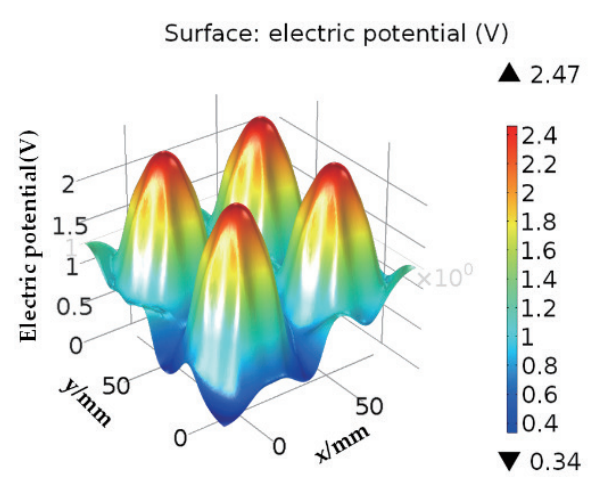

(a)

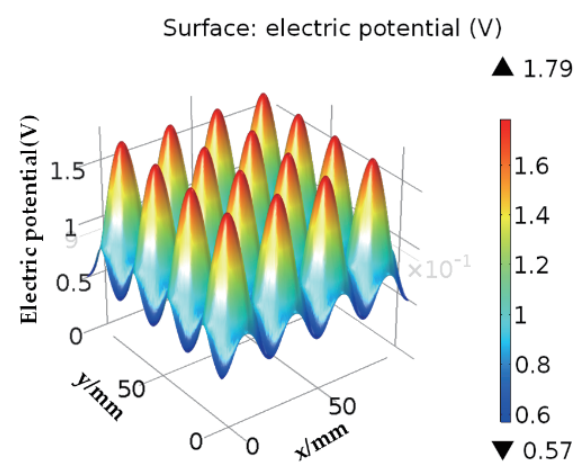

(b)

Fig. 9. (Color online) Potential distribution of the two array sensors: (a) $2 \times 2$ array sensor and (b) $4 \times 4$ array sensor. The potential of the sensor is distributed on the $X Y$ plane and the $Z$-axis at $5 \mathrm{~mm}$.

sensor because the $4 \times 4$ array has many sensors. The sensor capacitance component was inputted into Eq. (2), and the results are shown in Fig. 10.

Figure 10 shows that the sensitivity of the components of the two array sensors was basically the same and showed improved stability. The sensitivity of the $4 \times 4$ array sensor was slightly lower than that of the $2 \times 2$ array sensor, and the speed of decline was rapid because of the decrease in the single sensor measurement area and effective common edge length. The penetration performance of the sensor weakened, and the maximum thickness of the object required a certain sacrifice. Considering that the performance of the $4 \times 4$ array sensor involved a certain amount of sacrifice and many sensors were used, we adopted the $2 \times 2$ array arrangement of ice crystals as the electrode in the rainfall sensor (Fig. 11) to efficiently and accurately estimate rainfall.

Figure 11 shows the $2 \times 2$ array rainfall sensor used in this study. However, an array-type rainfall sensor involves many measurements in the process because of the increased number of sensors. An algorithm must be applied to carry the data fusion of multichannel measurement to obtain stable and accurate rainfall data. 


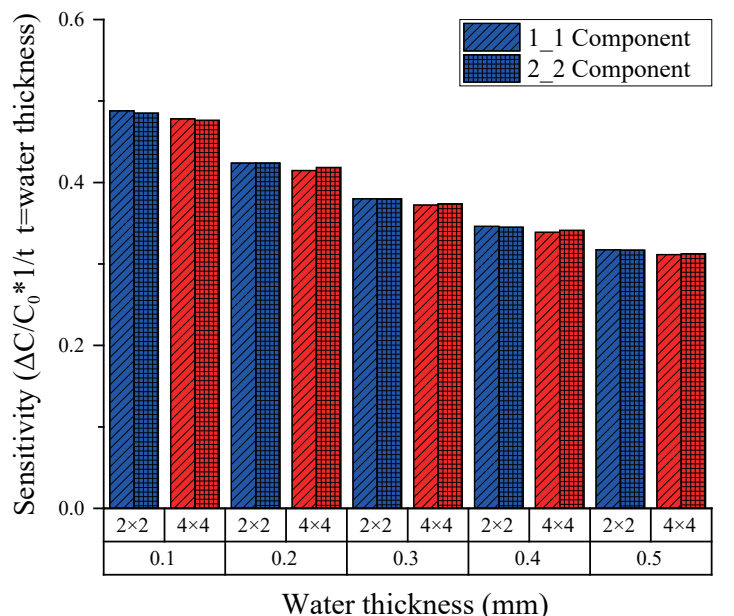

Fig. 10. (Color online) Sensitivity bar chart of the 2 $\times 2$ and $4 \times 4$ array rainfall sensors.

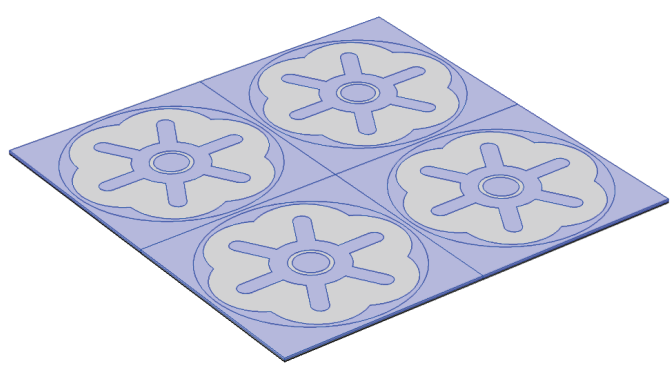

Fig. 11. (Color online) $2 \times 2$ array rainfall sensor.

\subsection{Data aggregation strategy for an adjacent capacitance array sensor}

When the data from a sensor array are aggregated, weighting is a common strategy adopted to obtain consistent and accurate measurement results. Several differences exist in the manufacturing process of each capacitance-measuring electrode, and each working environment differs. Under the same rainfall conditions, the water film thickness measured on each electrode deviates. This deviation can be corrected by a weighted aggregation method. In actual applications, however, falling rain is distributed unevenly on the surface of the sensor, and the actual thickness of the water film between electrodes constantly changes. Thus, a weighted data aggregation method with fixed weights is unsuitable for measurements obtained from a rainfall sensor array.

In this study, an adaptive weighted aggregation algorithm was used to update and aggregate multielectrode capacitance data. ${ }^{(27)}$ The accuracy of different measuring electrodes was evaluated by measuring the capacitance of each electrode dynamically. The weighting factor of each sensor was adjusted dynamically according to the accuracy of the evaluation results. High sensor accuracy in the process of determining the amount of rainfall had the highest weight. The algorithm identified the optimal weighting factor of each sensor in an adaptive manner and used the weighted factor to perform data aggregation on data acquired by multiple sensors.

We used the simulation data of the $2 \times 2$ array sensor in different environments as an example to illustrate the specific implementation of the program. The expected simulation state is to judge the state of the $3.8 \mathrm{~mm}$ acrylic plate covering the $0.5 \mathrm{~mm}$ water film on the surface. Under this condition, the average of a group of capacitances was obtained, and the relative percentage (relative to the dry state) was calculated as the true value ( $X=15.85 \%$ ). The simulation models presented in Sect. 3.2 were then utilized to simulate 10 geometric interference environments. The relative percentage of 10 sets of measured values was obtained by simulation, as shown in Table 4 . 
Table 4

Increasing percentage of each sensor component.

\begin{tabular}{ccccc}
\hline Groups & 1_1 Component & 2_2 Component & 3_3 Component & 4_4 Component \\
\hline 1 & 15.97 & 15.97 & 15.98 & 15.99 \\
2 & 16.01 & 16.04 & 16.00 & 16.00 \\
3 & 16.02 & 16.03 & 16.01 & 15.99 \\
4 & 15.92 & 0.23 & 15.93 & 15.81 \\
5 & 15.81 & 15.93 & 11.34 & 15.92 \\
6 & 15.87 & 15.88 & 13.79 & 13.76 \\
7 & 15.86 & 15.89 & 13.80 & 13.77 \\
8 & 15.82 & 17.68 & 15.82 & 15.80 \\
9 & 15.82 & 17.72 & 13.74 & 15.84 \\
10 & 15.87 & 13.80 & 13.78 & 15.89 \\
\hline
\end{tabular}

In Table 4, the relative percentage of each sensor measurement under the environment of each sensor array was. For the unbiased estimation of the true value of $X$, the variance of each sensor was $\delta_{1}^{2}, \delta_{2}^{2}, \delta_{3}^{2}$, and $\delta_{4}^{2}$. The weighted factors were $W_{1}, W_{2}, W_{3}$, and $W_{4}$. The estimated values after aggregation and the weighting factors meet the following conditions.

$$
\begin{gathered}
\hat{X}=\sum_{i=1}^{4} W_{i} x_{i} \\
\sum_{i=1}^{4} W_{i}=1
\end{gathered}
$$

Given that the sensors are independent of each other, the true value of $X$, which is an unbiased estimation, is equivalent to

$$
\delta^{2}=E\left[\sum_{i=1}^{4} W_{i}^{2}\left(X-x_{i}\right)^{2}\right]=\sum_{i=1}^{4} W_{i}^{2} \delta_{i}^{2} .
$$

According to the above equation, the total mean square error is a multivariate, two-function weighted factor of each sensor. Thus, the total mean square error must have the maximum value. The solution of the minimum value must satisfy Eq. (4)'s constraint condition for the multivariate function. With the multivariate-function extreme-value method, we determined the corresponding weighted factor when the total mean square error was minimum as follows. ${ }^{(28)}$

$$
W_{i}=\frac{1}{\delta_{i}^{2} \sum_{j=1}^{4} \frac{1}{\delta_{j}^{2}}}, i=1,2,3,4
$$

In the above equation, the variance can be obtained through various methods as follows.

$$
\delta_{i}^{2}=E\left[\left(x_{i}-\bar{x}\right)^{2}\right], i=1,2,3,4
$$


The average value for $x_{i}$ is $\bar{x}$, and the corresponding minimum mean square error is

$$
\delta_{\min }^{2}=\frac{1}{\sum_{i=1}^{4} \delta_{i}^{2}}
$$

The results of the array capacitive sensor were obtained by inputting the simulation results into Eqs. (6) and (3). Figure 12 shows that the result was a relative percentage of the unbiased estimates of the array capacitive sensor. On the basis of the mapping rules between the measured results and rainfall, data can be converted to rainfall data, thus completing the measurement of rainfall.

\section{Rainfall Sensor Experiment}

\subsection{Construction of an experimental platform for rainfall sensor measurements}

An experimental sensor platform was designed to evaluate the performance of the rainfall sensor array described in this paper. The experimental platform comprised a capacitance digital conversion module, a rainfall sensor, an insulating medium, and a simulated rainfall platform. The capacitance digital conversion module used the FDC1004EVM evaluation kit produced by Texas Instruments, Inc. (Dallas, TX, USA), with four measurement channels in a plug-andplay system corresponding to four shielding channels. ${ }^{(29)}$ The full range of each measurement channel was $+15 \mathrm{pF}$, and the resolution reached $0.5 \mathrm{fF}$. The rainfall sensor was the $2 \times 2$ array proximity capacitive sensor described in this paper. The manufacturing cost of the sensor was low. Thus, we used PCB, and the electrode material was copper. Each sensor in the array corresponded to a measurement channel of the digital conversion module. The rain sensor was placed snugly beneath a $3.8 \mathrm{~mm}$ acrylic plate that served as an insulating medium and provided good waterproofing. The rainfall simulation platform used a shower nozzle with a variablespeed pump to simulate rainfall; by adjusting the pump flow, various amounts of rainfall were simulated. The entire rainfall sensor measurement system is shown in Fig. 13.

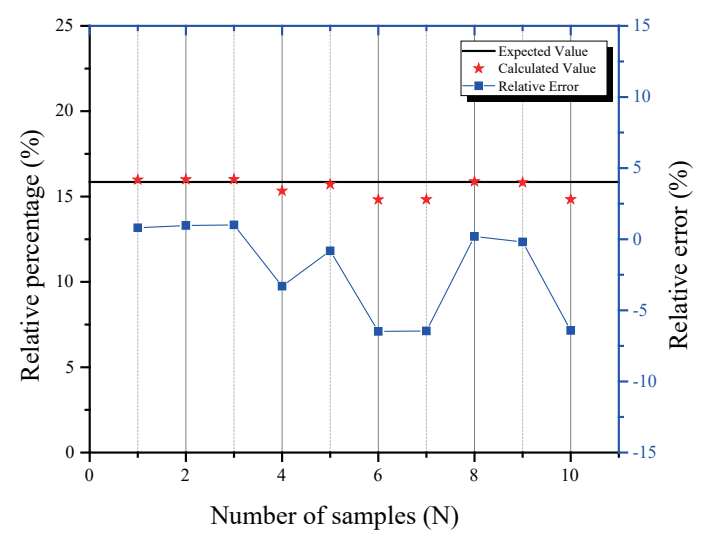

Fig. 12. (Color online) Aggregation results of $2 \times 2$ array sensor data. 


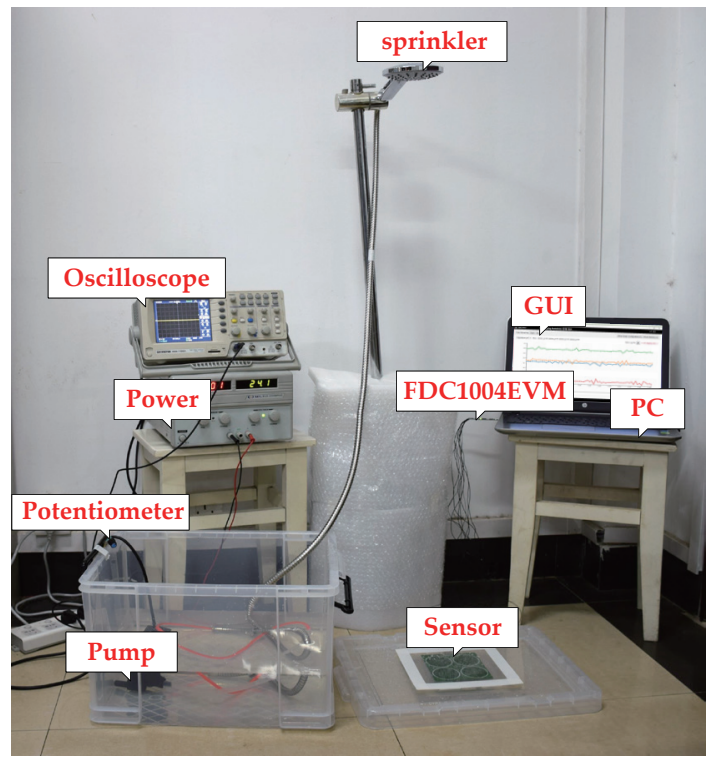

Fig. 13. (Color online) Actual picture of system hardware.

Meteorological departments generally define rainfall intensity as rainfall within a unit of time. The average of the capacitance measurements under different rainfall intensities must be obtained and used as the reference value to evaluate the various indices of the rainfall sensor. Different simulated rainfall intensities can be obtained by regulating the pump's control voltage because a speed-regulating water pump provides different water flow rates under different control voltages. The experiment platform was realized easily by the method of artificial precipitation simulation. The power supply of the platform can be accurately adjusted to overcome the problems associated with the previous use of the valve to adjust the water flow, such as difficulty of operation, poor repeatability, and so on. ${ }^{(30)}$ These changes are demonstrated in the following experiments.

After building the experimental platform, we referred to the rainfall intensity levels defined by the local meteorological department. Pump voltage was adjusted accordingly. The corresponding measurement channel capacitances were obtained, and the data were aggregated. In addition, the sensor was calibrated because of the existence of a cable, which could lead to errors in capacitance measurement. In the calibration process, a median filter was used to remove the spike pulse in the sampling process. The original and error values were utilized as the reference values of the sensor when dry. After calibration, the capacitance reference values of sensor channels 1-4 when dry were 3.8998, 4.3536, 3.9857, and $3.3984 \mathrm{pF}$, respectively. The average value of rainfall at different voltages was calculated according to these values, as shown in Table 5. In addition, the water supply was directly proportional to the supply voltage, the rainfall in the light rain is less than $10 \mathrm{~mm} / \mathrm{h}$, the rainfall in the moderate rain is about 10 to 25 $\mathrm{mm} / \mathrm{h}$, the rainfall in the heavy rain is about 25 to $50 \mathrm{~mm} / \mathrm{h}$. The supply voltage of the platform could be freely selected but should not be less than $1.5 \mathrm{~V}$; otherwise, it might not drive the platform rainfall. 
Table 5

Rainfall rank from experimental results using the simulated rainfall platform.

\begin{tabular}{lccccc}
\hline Rainfall rank & Voltage (V) & Channel 1 & Channel 2 & Channel 3 & Channel 4 \\
\hline Light rain & 1.5 & $4.5223^{*}$ & 4.9527 & 4.667 & 4.1618 \\
Moderate rain & 2.0 & 4.5525 & 5.0638 & 5.0115 & 4.3917 \\
Heavy rain & 3.0 & 4.6354 & 5.1995 & 5.2076 & 4.5359 \\
\hline
\end{tabular}

*Average value; true value of fringing capacitance $(\mathrm{pF})$.

After obtaining the reference data, the average value of the four channels was calculated, and the value of the three groups was obtained as the expected value of different amounts of rainfall. The performance indices of the rainfall sensor described in this paper were tested and evaluated using the platform.

\subsection{Rainfall measurement experiments}

The performance indices of the rainfall sensor included accuracy, repeatability, stability, and dynamic characteristics. The performance indices of the designed capacitor-type rainfall sensor were tested by using the experimental platform and related data obtained in advance.

The power supply voltage of the platform was increased at the same frequency, that is, under the same conditions, and three groups of data were obtained by repeated experiments to verify the repeatability and stability of the sensor. The same frequency change in a short period was used to repeat the experiment. Fifteen groups of samples were selected, and the average value and standard deviation of each sample were calculated. Figure 14 shows the results of experiments. The aggregated results of the sensor capacitance under different voltages and rainfall evaluation results were obtained and compared with the expected values by adjusting the control voltage input of the pump controller and changing the flow rate of the pump. Figure 15 shows the results of experiments. Moreover, the control voltage of fixed-speed pumps and the signal line of the single channel of the array-type rainfall sensor were removed to verify the reliability of the rain sensor. Then, the measurement results under different conditions were compared. The results of the experiments are shown in Table 6 .

The dynamic characteristic of a rainfall sensor is also an important index. In this study, the input voltage of the variable-speed pump was connected to the signal generator, and the signal generator voltage was set to a frequency of $0.1 \mathrm{~Hz}$. Real-time capacitive sensor and rainfall measurement results were recorded, and the signal characteristics of the measurement results were observed. The results are shown in Fig. 16.

\subsection{Results of experiments}

Figure 14(a) shows that the sensor demonstrated good stability over a long period. Figure 14(b) shows that the error bar was short, which meant that the standard deviation of the measurement results was small. Therefore, the results of the repeated measurements of the sensor demonstrated good consistency and repeatability. 


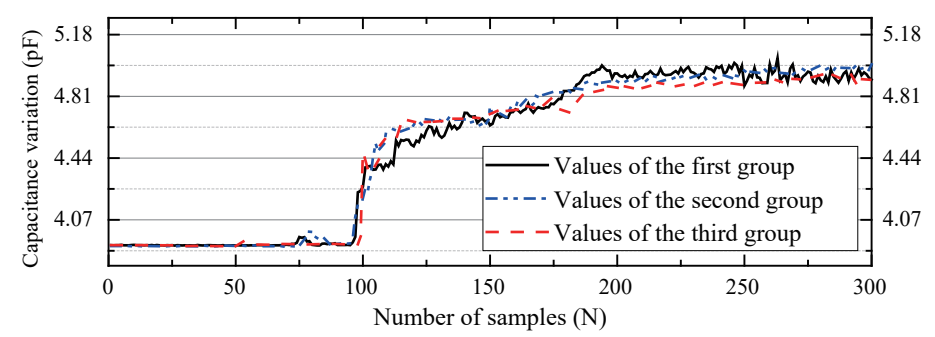

(a)

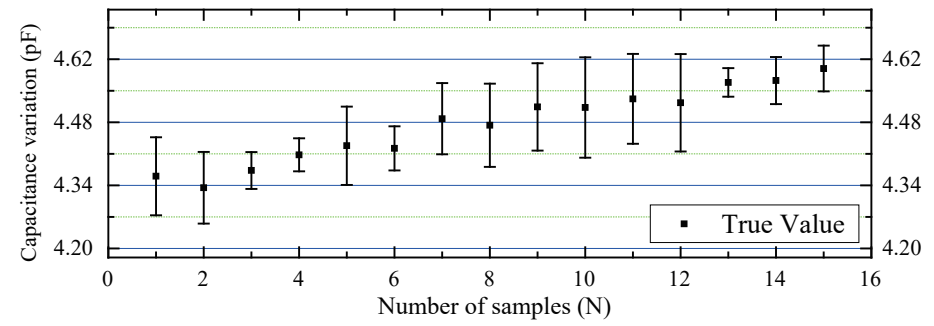

(b)

Fig. 14. (Color online) Repeatability and stability of the sensor. (a) Three groups of capacitance variation results in the same conditions and (b) average and standard deviation of the 15 groups of samples.

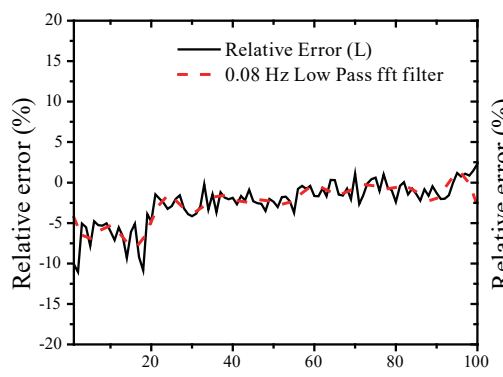

(a)

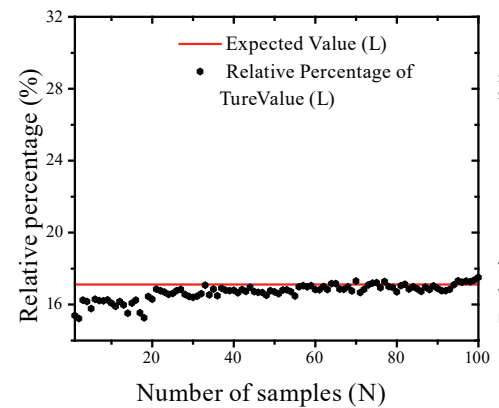

(d)

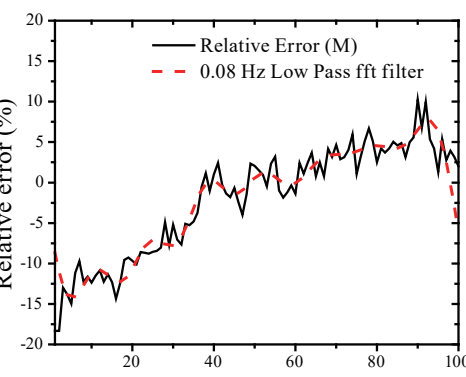

(b)

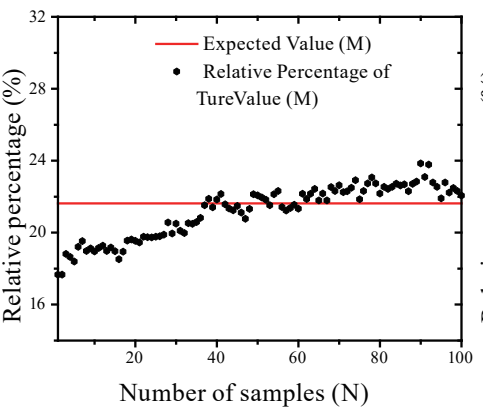

(e)

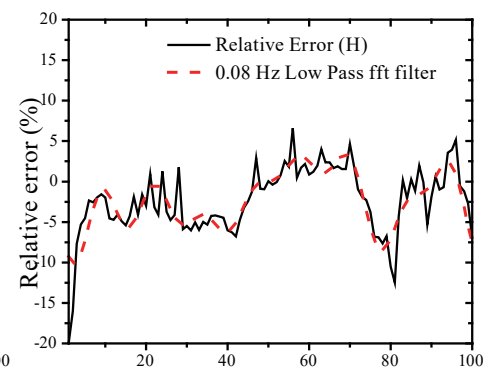

(c)

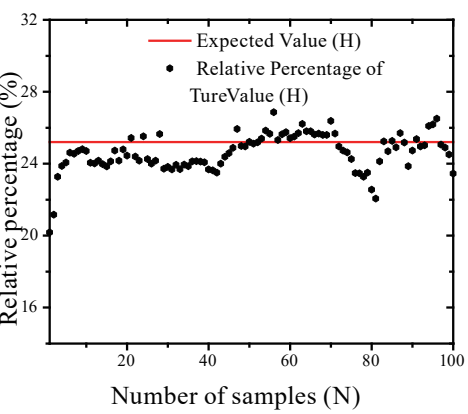

(f)

Fig. 15. (Color online) Relative error of the rainfall sensor: (a) light rain, (b) moderate rain, and (c) heavy rain. Results of aggregating the measurement data: accuracy based on conditions of (d) light rain, (e) moderate rain, and (f) heavy rain.

Table 6

Rainfall measurement results.

\begin{tabular}{lc}
\hline Rainfall & Probability (\%) \\
\hline Light rain & $60^{*}$ \\
Moderate rain & 76 \\
Heavy rain & 84 \\
\hline
\end{tabular}

*Percentage of approximately agreed parts of true values and expected values. 
(a)

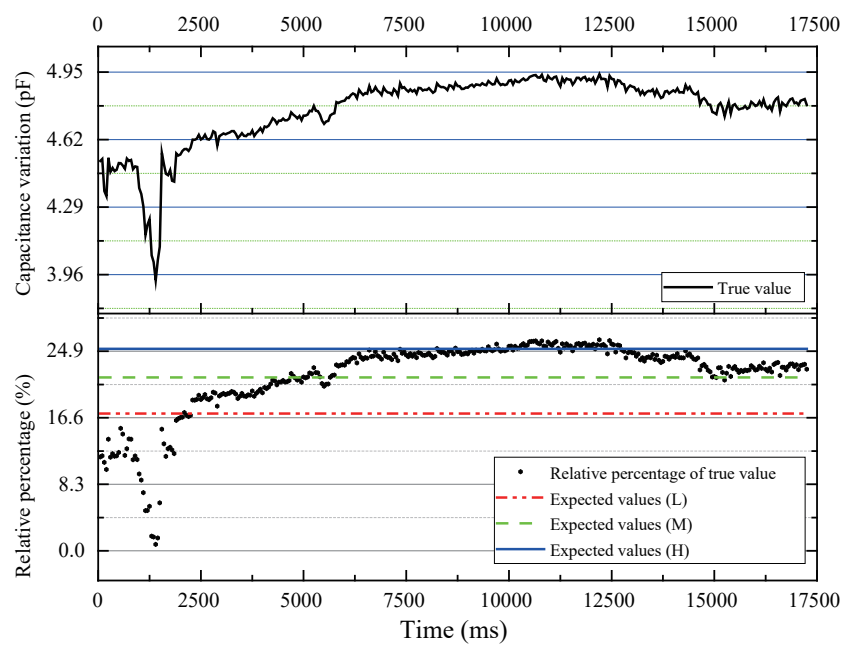

Fig. 16. (Color online) (a) True value of the rainfall sensor in real time and (b) measurement result of the rainfall sensor.

Figure 15 shows that the sensor capacitance measurement and expected values agree under the same control voltage. Figure 15 also shows the relative error of the sensor after using a fast Fourier transform (FFT) filter.

The average measurement error of the sensor was calculated according to the relative error value of different rainfall amounts in Fig. 15, as shown in Table 7. Table 7 shows that the maximum measurement error for different rainfall values of this sensor was only $\pm 2.48 \%$, whereas the measurement error of other sensors in the market is about $\pm 3 \%$. Therefore, the rainfall sensor designed in this study has higher accuracy than other sensors in the market. In the actual application process, sensors whose parameters have been calibrated produce an accurate assessment of the amount of rainfall achievable.

Table 6 shows the results of one of the cases, in which the signal line of a channel is removed to verify whether the array sensor can still accurately determine the amount of rainfall. The results presented in Table 6 show that although some electrode wear or occlusion occurred, the array-type sensor still obtained accurate rainfall measurements when the rainfall sensor had multiple channels.

Figure 16 shows that the sensor can measure and judge the change in rainfall in real time, and it proves that the sensor possesses good dynamic characteristics and can be used in practical applications. Manufacturers can rely on the sensors designed in this study to provide customers with ideal solutions.

The above experiments were repeated after changing the penetration medium thickness of the rainfall sensor. Observation of the performance of the rainfall sensor revealed that when the thickness of the medium was greater than a certain value (about $6 \mathrm{~mm}$ ), the performance of the sensor decreased dramatically, and the rainfall measurement results could not be evaluated correctly.

The experimental results indicated that the array-type rainfall sensor designed in this study has high sensitivity and reliability, can make a correct assessment of the amount of rainfall 
Table 7

Measurement error of sensors.

\begin{tabular}{lccc}
\hline Rainfall & Light rain (\%) & Moderate rain (\%) & Heavy rain (\%) \\
\hline Rainfall sensor & $\pm 2.48^{*}$ & \pm 2.04 & \pm 2.35 \\
Other sensors & $\pm 3^{* *}$ & & \\
\hline
\end{tabular}

*Measurement error for different rainfall values of this rainfall sensor.

${ }^{* *}$ Measurement error of other capacitance rainfall sensors.

on a surface of the detecting medium with a certain thickness, and possesses good dynamic characteristics.

\section{Conclusions}

An ice crystal sensor with the best performance is obtained by comparing the three types of sensor. The insulation medium penetration of the described rainfall sensor was significantly improved by optimizing the design of the capacitor electrode, and high sensitivity to water film thickness was obtained. Unbiased estimation of rainfall data was realized using a multicapacitor array arrangement and a multisensor data aggregation algorithm. In the future, we will also make a further research and analysis of factors that affect the performance of the sensor, for example, the sensitivity of the sensor will be decreased when the sensor is covered with iron overlay, if it is integrated in a structural material. We will pay more attention to other cross sensitivities on temperature and dirt contamination.

Rainfall sensors are widely used in weather monitoring, modern agriculture, the automobile industry, intelligent home functionality, and so on. The installation method, cost, and precision of rainfall sensors used in different application scenarios are different. In this study, an array of proximity capacitive rain sensors was designed on the basis of the principle of proximity capacitance to conduct real-time noncontact measurement with the surface of an insulating medium. The sensor array demonstrates a certain measurement accuracy, but its installation method and usage in cost-sensitive applications are restricted. The program and ideas used in the design of the rain sensor described in this paper can provide a reference for the design of similar sensors.

\section{Acknowledgments}

This research is supported by the National Natural Science Foundation of China (61663011) and the postdoctoral fund of Jiangxi Province (2015KY19).

\section{References}

1 X. B. Sun, D. S. Wang, H. Liu, and G. H. Hou: Instr. Tech. Sens. 49 (2010) 1256.

2 Ford Scorpio: Automatic wiper for the Ford Scorpio 95+. http//www.fordscorpio.co.uk/autowiper.htm (accessed October 2016).

3 S. Mcloone, P. Hung, and G. Irwin, and R. J. Kee: IEEE Sens. J. 6 (2006) 1627. 
4 T. Iuchi and A. Gogami: Rev. Sci. Instrum. 80 (2009) 286.

5 V. D. P. Onana, E. Trouve, G. Mauris, and J. P. Rudant: Geosci. Remote Sens. IEEE Trans. 41 (2003) 2540.

6 P. Klokoc, I. Lujo, M. Bosiljevac, and N. Burum: Elmar-2008. Int. Symp. 49 (IEEE, 2008) 625.

7 C. Gaber, K. Chetehouna, H. Laurent, and C. Rosenberger: IEEE Int. Symp. Industrial Electronics (IEEE, 2008) 1120.

8 P. Y. Chen, C. C. Chen, W. K. Yeh, Y. Chang, D. C. Huang, S. S. Yu, C. S. Tsai, Y. J. Huang, W. C. Lin, S. I. Chu, P. Lon, T. H. Lin, and S. C. Liu: J. Sens. (2014) Article ID 238350. doi: $10.1155 / 2014 / 238350$

9 Y. Kojima, R. Shigeta, N. Miyamoto, Y. Shirahama, K. Nishioka, M. Mizoguchi, and Y. Kawahara: Sensors 16 (2016) 1292. doi: $10.3390 / \mathrm{s} 16081292$

10 A. Ramezani Akhmareh, M. Lazarescu, O. Bin Tariq, and L. Lavagno: Sensors 16 (2016) 1448. doi: $10.3390 / \mathrm{s} 16091448$

11 M. L. Morrissey, H. J. Diamond, M. J. Mcphaden, H. P. Freitag, and J. S. Greene: J. Atmos. Oceanic Technol. 29 (2012) 834.

12 W. Sa-Ngiamvibool, N. Angkawisittpan, A. Nuan-On, C. Photong, and A. Kangrang: Int. J. Eng. Technol. 5 (2013) 3596.

13 J. A. Nystuen: J. Atmospheric Oceanic Technol. 16 (1999) 1025.

14 A. Troiano, E. Pasero, and L. Mesin: IEEE Trans. Instrum. Meas. 60 (2011) 1091.

15 N. Li, B. Guo, and P. Wang: Chin. J. Mech. Eng. 46 (2010) 12.

16 L. Zhang, S. Ye, S. Zhou, and F. Liu: Proc. 2016 4th Int. Conf. Electrical \& Electronics Engineering and Computer Science. (ICEEECS, 2016) 737.

17 B. Lai, Z. Deng, X. Wang, and J. Yan: Proc. 2016 Int. Conf. Education, Management, Computer and Society (EMCS, 2016) 1581.

18 I. Bord, P. Tardy, and F. Menil: Sens. Actuators, B 114 (2006) 640.

19 W. Sa-Ngiamvibool, N. Angkawisittpan, A. Nuan-On, C. Photong, and A. Kangrang: Int. J. Eng. Technol. 5 (2013) 3596.

20 Y. Han: Design and Optimization of Proximity Capacitive Sensor Based on Insulating Film Measurement. (Doctoral dissertation, Xidian University, 2013).

21 C. G. Xie, A. L. Stott, A. Plaskowski, and M. S. Beck: Meas. Sci. Technol. 1 (1990) 65.

22 L. Peng, J. Ye, G. Lu, and W. Yang: Sens. J. IEEE 12 (2013) 1554.

23 J. G. Webster: IEEE Electr. Insulation Mag. (1999) 87.

24 N. Li and Y. Han: J. Southwest Jiaotong University 2 (2014) 330.

25 R. K. Wangsness: Electromagnetic Field (Wiley-VCH, 1986) p. 608.

26 H. Cao, E. Dong, Z. Fan, and Y. Pang: Comput. Meas. Control 21 (2013) 1711.

27 Y. Zhai and Y. Dai: Acta Metrologica Sinica 19 (1998) 69.

28 Y. Liang, L. Xu, and B. Zhu: Comput. Meas. Control 17 (2009) 1447.

29 Texas Instruments: FDC1004 4-Channel Capacitance-to-Digital Converter for Capacitive Sensing Solutions, (Rev. B), http://www.ti.com/lit/ds/snoscy5b/snoscy5b.pdf (accessed October 2016).

30 Z. Tao: Research on infrared rain sensor and its test platform. (Doctoral dissertation, Shanghai University, 2009). 